\title{
Comparison of LDL- Cholesterol Enzymatic Method with Friedewald's Formula
}

\section{Hamidreza Yazdi}

Pharmacist, Faculty of Medicine, Golestan University of Medical Sciences, Gorgan, Iran

Fatemeh Piran (BSc)

Laboratory Sciences Technologist, Sayad Shirazi Hospital, Golestan University of Medical Sciences, Gorgan, Iran

Sanaz Royani (MSc)

Department of Health Policy, School of

Health Management and Information

Sciences, Iran University of Medical

Sciences, Tehran, Iran

Mojgan Nejabat (MSc)

Department of Medicinal Chemistry, Laboratory Science Research Center,

Mashhad University of Medical Sciences, Mashhad, Iran

Gholamreza Roshandel (PhD)

Department of

Epidemiology, Gastroenterology and Hepathology Research Center, Golestan University of Medical Sciences, Gorgan, Iran

Mahsa Taherizadeh(MSc)

Department of Clinical Biochemistry,

Biochemistry \& Metabolic Disorders

Research Center, Golestan University of

Medical Sciences, Gorgan, Iran

Hamid Reza Joshaghani (PhD)

Department of Biochemistry, Laboratory

Science Research Center, Golestan

University of Medical Sciences, Gorgan,

Iran

Corresponding author: Hamidreza Joshaghani

Email: Joshaghani@goums.ac.ir

Tel: +981732443563

Address: Department of Biochemistry,

Laboratory Science Research Center,

Golestan University of Medical Sciences,

Gorgan, Iran

Received: 13 oct 2015

Revised: 11 Nov 2015

Accepted: 15 Nov 2015
ABSTRACT

Background and Objective: Concentration low-density lipoprotein (LDL) is one of the strongest indicators of atherosclerosis and predicts the diagnosis of cardiovascular diseases. LDL measurement accuracy is very important. LDL can be measured directly, such as enzymatic and nephelometry methods or can be calculated using Friedewald's formula. Despite the development of enzymatic methods and LDL nephelometry still in most laboratories is calculated using Friedewald's formula. The aim of this study was an investigation of correlation coefficient between two methods of measuring LDL- cholesterol levels.

Methods: This descriptive cross-sectional study, performed on the 1141 patients. Cholesterol, triglycerides, HDL, LDL all patients assayed by enzymatic method. For patients with triglyceride levels of less than $400 \mathrm{mg} / \mathrm{dl}$ had LDL levels were calculated by Friedewald's formula. Normal levels of LDL/HDL ratio of less than 3.5 were considered.

Results: 0f the 1141 patients participating in this study, $38.3 \%$ men and $61.7 \%$ women. The mean patient age was $46.3 \pm 16.1$ years. Mean serum cholesterol, triglycerides and HDL were $177.9 \pm 41.1,132.9 \pm 73.2$ and $45.8 \pm 13.2 \mathrm{mg} / \mathrm{dl}$, respectively. Average direct and calculated LDL concentration was $02.1 \pm 23.1$ and $105.5 \pm 35.8$, respectively. The direct measurement of LDL, LDL/HIDL levels in $97.1 \%$ of cases was normal, while $85.1 \%$ of the calculation of LDL were normal. Pearson correlation coefficients were obtained by two methods: $0.869(\mathrm{p}<0.001)$.

Conclusion: Despite the favorable correlation between two methods of measurements of LDL, the results of a calculation method is more than direct method. This can have a negative impact on the judgment of the treating physician.

Keywords: LDL, Enzymatic Method, Friedewald's Formula.

This paper should be cited as: Yazdi Hr, Piran F, Royani S, Nejabat M, Roshandel G, Taherizadeh M, Joshaghani HR [Comparison of LDL-Cholesterol Enzymatic Method with Friedewald's Formula]. mljgoums. 2015; 9(4):54-56 


\section{INTRODUCTION}

The Cause many deaths worldwide is coronary artery disease and many studies have shown that the risk of these diseases are related to increase of level low-density lipoprotein (LDL) (1). Lipoproteins carry cholesterol in plasma is five classes, including HDL, LDL, VLDL, IDL and LDL (2). LDL concentration is a marker in the diagnosis and prediction of cardiovascular disease. Also, studies have shown an association between ischemic and LDL is more than total cholesterol (3-6). LDL measurement accuracy is very important. Usually, total cholesterol and HDL-c measured directly and LDL-C calculated by Friedewald's formula (FF). The accuracy of the results of FF depends on parameters such as total cholesterol, TG and HDL. In this method, the TG exceeds from $4.5 \mathrm{mmol} / \mathrm{L}$ or presence of abnormal lipoprotein, differences can be seen between calculated LDL with reference method. Reference method for measuring LDL-C is a beta quantification method that's based on a twostep, ultracentrifugation and precipitation. LDL can be measured by chromatography, electrophoresis, immunological and nephelometry methods or can be calculated by FF that is a simple, convenient and low cost method $(1,4,9,10)$. In this method (FF), LDL is calculated by this equation, $\mathrm{LDL}=$ total cholesterol _ (VLDL-C + HDL-C) [5], but this formula when used for the TG less than 400 $\mathrm{mg} / \mathrm{dl}$. But this formula is used when the TG < $400 \mathrm{mg} / \mathrm{dl}$, so factors such as concentrations of TG more than $400 \mathrm{mg} / \mathrm{dl}$ and initial hyperlipidemia type IV is the main limiting uses of FF $(5,11)$. This formula for patients with diabetes, hepatopathy and nephropathy even when the TG is less than $400 \mathrm{mg} / \mathrm{dl}$ is not used (1). Despite its limitations, this method typically used to estimate the concentration of LDL-C in the laboratory (12) some studies have shown that LDL calculated using the FF in patients with TG between 4.51 and $8.82 \mathrm{mmol} / 1$ did not show any significant error. LDL-C was calculated using the FF even in patients with high TG $4.5 \mathrm{mmol} / 1$ can be valid (5). In most studies, calculated LDL-C was slightly lower than the direct method (4). The aim of this study was to evaluate the correlation coefficient of LDL measured enzymatic with the FF.

\section{MATERIAL AND METHODS}

This cross-sectional descriptive-analytical study performed on 1411 patients. Cholesterol, triglycerides, HDL, LDL (Pars Azmoon) all patients assayed by enzymatic method by autoanalyzer (Mindray BS-200). For patients with triglyceride levels of less than $400 \mathrm{mg} / \mathrm{dl}$ had LDL levels were calculated by Friedewald's formula. Normal levels of LDL/HDL ratio of less than 3.5 were considered. A significance level of $95 \%$ for all tests was considered.

\section{RESULTS}

People participated in this study were $38.3 \%$ male and $61.7 \%$ female. The mean (SD) age was 46.3 (16.1) years. The mean (SD) serum cholesterol, triglycerides and HDL were 177.9 (41.1), 132.9 (73.2) and 45.8 (13.2) $\mathrm{mg} / \mathrm{dl}$, respectively. The mean (SD) LDL concentration was 82.1 (23.1) and 105.5 (35.8) $\mathrm{mg} / \mathrm{dl}$ with direct assay method and calculation methods, respectively $(\mathrm{p}=0.001)$. The LDL levels in the direct measurement were normal in $89.3 \%$, and in the calculation method $99.8 \%$ of the cases were normal $(P=0.001)$. Serum LDL levels are measured using a direct method significant positive correlation with serum LDL levels were measured using a calculation method (Figure 1).

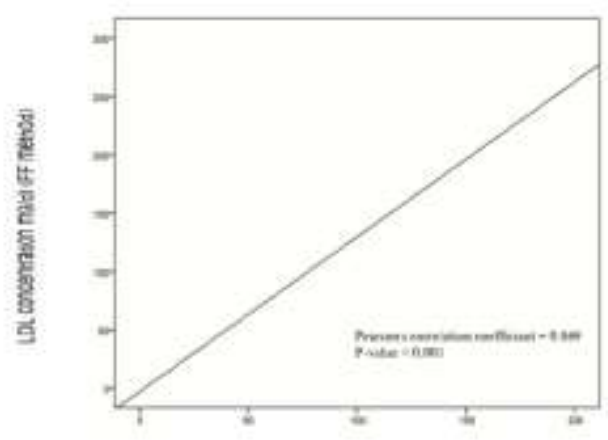

Figure 1- Correlation coefficient between the two methods of measuring LDL 


\section{DISCUSSION}

Since heart disease is the most common cause of mortality worldwide and its increase is proportional to the increase in LDL, the measurement accuracy of LDL is essential. There are various methods for measuring LDL, which routinely in laboratories LDL is calculated from the Friedewald's method, but this method has limitations that the more important it is level of TG above $400 \mathrm{mg} / \mathrm{dl}$. Some studies have shown when the TG higher than 400 this method has not valid. As an example, a study of Balal and colleagues on 193 patients show when the TG less than $400 \mathrm{mg} / \mathrm{dl}$ can use from FF (4). Also Türkalp study on 47 patients showed a high correlation between the measurement of LDL and FF a direct method $(12,13)$. Another study by Cordova and coworkers with the aim of comparing the two methods of measuring LDL (direct method and FF) on 10664 patients was performed. They showed that the samples with different concentration TG two methods were not similar functional. When TG less than $150 \mathrm{mg} / \mathrm{dl}$, may be LDL calculated by the FF can be invoked, but if TG between $301-400 \mathrm{mg} / \mathrm{dL}$ cannot be recommended to use of the FF (1). Another study by the same objective in 2005 reported even when TG less than $200 \mathrm{mg} / \mathrm{dl}$ is between LDL obtained from both direct and FF significant differences exist and the correlation coefficient between the two methods was 0.88 , but some of the results inconsistent achieved and demonstrated that even in patients with TG more than $4.5 \mathrm{mmol} / \mathrm{l} \mathrm{FF}$ method is valid and usable (14). A study by Mora and colleagues in 2009 of 27,331 healthy women with TG less than 400 $\mathrm{mg} / \mathrm{dl}$ was performed. Baseline LDL determined by $\mathrm{FF}$ and direct measurement of fasting and non- fasting were compared homogenous risk (CVD) CVD in during an 11 year period. While low concentrations of LDL indirect method may be causing a lot of people be classified wrong in lower NCEP (Cholesterol Education Program

\section{REFERENCES}

1. Mendes de Cordova CM, Schneider C, Juttel L, Mendes de Cordova M. Comparison of LDL-Cholesterol Direct Measurement with the Estimate Using the Friedewald Formula in a Sample of 10,664 Patients. Arquivos Brasileiros de Cardiologia. 2004; 83(6): 482-7.

2. Jabbar J, Siddiqui I, Raza Q. Comparison of two methods (Precipitation Manual and Fully Automated Enzymatic) for the Analysis of HDL and LDL Cholesterol. JPMA. 2006; 56(2): 59-61.
International). In addition, the lack of association between non-fasting direct LDL with CVD ( $P<0.0001$ ), causes a lot of questions regarding the clinical utility of direct methods for LDL with non-fasting blood samples (1517). Can and colleagues' study was conducted on 1001 patients, cholesterol and TG levels were measured by enzymatic methods, and HDL and LDL levels were measured using the direct method was performed and showed LDL was estimated by the Friedewald's method significantly correlated with the direct method (P <0.01) (18). Study Timón-Zapata et al to study the effects of high levels, HDL in the calculation LDL use FF and other formulas that have recently been proposed, in the 2603 samples the HDL less than $20 \mathrm{mg} / \mathrm{dl}$ and 1953 samples with HDL more than $100 \mathrm{mg} / \mathrm{dl}$ done, showed significant differences encirclement between LDL was calculated with the formula and the direct method with two levels of HDL-C there. The results of the analysis suggest that none of the formulas should be used to calculate LDL in samples with high concentrations of HDL because there is no direct correlation with LDL may be used (19).

\section{CONCLUSION}

Despite the favorable correlation between measurement methods of LDL, the results of a calculation method is more than direct measure, this can have a negative impact on the judgment of the treating physician. The lack of standardized methods for direct measurement and comparison of results in different levels of $\mathrm{TG}$, cholesterol, and HDL seems to require a comprehensive study and grouped based on different values of TG and HDL.

\section{ACKNOWLEDGMENT}

We thank the Kavosh medical laboratory that helped us in this project.

\section{CONFLICT OF INTEREST}

The authors declare no conflict of interest between them.

3. Vujovic A, Kotur-Stevuljevic J, Spasic S, Bujisic N, Martinovic J, Vujovic M, et al. Evaluation of different formulas for LDL-C calculation. Lipids Health Dis. 2010; 9: 27. doi: 10.1186/1476-511X-9-27.

4. Balal M, Paydas S, Inal T, Demir E, Kurt C, Sertdemir Y. Validation of the Friedewald formula for the determination of low-density lipoprotein cholesterol in renal transplant recipients. Renal Failure. 2010; 32(4):4558. doi: 10.3109/08860221003658266. 
5. Tremblay AJ, Morrissette H, Gagné JM, Bergeron J, Gagné C, Couture P. Validation of the Friedewald formula for the determination of low-density lipoprotein cholesterol compared with beta-quantification in a large population. Clinical Biochemistry. 2004; 37(9): 785-790.

6. Yamashita S, Nakamura M, Koizumi H, Oku H, Joes C. Sandoval J, et al. Evaluation of a Homogeneous Assay for Measuring LDL-cholesterol in Hyperlipidemic Serum Specimens. J Atberoscler Tbromb. 2008; 15(2): 82-86.

7. Dong J, Guo H, Yang R, Li H, Wang S, Zhang J, et al. Serum low-density lipoprotein and high-density lipoprotein cholesterol determined by ultracentrifugation and highperformance liquid chromatography. Clinica Cimica Acta. 2011; 52(2): 383-388: 1071-1076. doi: 10.1194/jlr.D008979.

8. Assmann G, Jabs H-U, Nolte W, Schriewer H. Precipitation of LDL with Sulphopolyanions: A Comparison of Two Methods for LDL Cholesterol Determination. J. Clinal Biochemistry. 1984; 22(11): 781785.

9. Pisani T, Pickering SW, DeLuca L, Gebski CP. Performance of a direct LDL-cholesterol method compared to beta quantification. Pure \&App Chern. 1996; 68(10): 1887-1892.

10. Bayer P, Veinberg F, Couderc R, Cherfils C, Cambillau $\mathrm{M}$, Cosson $\mathrm{C}$, et al. Multicenter evaluation of four homogenous LDL-cholesterol assays. Ann Biol Clin (Paris) 2005; 63(1): 27-41.

11. Esteban-Salan M, Aguilar-Doreste JA, Luisa M, ranzPena R, Juve-Cuxart S , Gich-Salarich I, et al. Multicentric evaluation of the homogeneous LDL-cholesterol Plus assay: Comparison with beta-quantification and Friedewald formula. Clinical Biochemistry. 2008; 41(1617): 1402-1409.

12. Türkalp I, Çil Z, Özkazanç D. Analytical performance of a direct assay for LDL-cholesterol:a comparative assessment versus Friedewald's formula. Anadolu Kardiyol Derg. 2005; 5: 13-17.
13. Schertman G, Patsches M, Sasse EA. Variability in cholesterol measurements: Comparison of calculated and direct LDL cholesterol determinations. Clinical Chemistry. 1996; 42(5): 732-737.

14. Sahu S, Chawla R, Uppal B. Comparison of two methods of estimation of low density lipoprotein cholesterol, the direct versus friedewald estimation. Indian Journal of Clinical Biochemistry. 2005; 20(2): 54-61. doi: 10.1007/BF02867401.

15. Evans SR, Fichtenbaum CJ, Aberg JA; A5087 Study Team.Comparison of Direct and Indirect Measurement of LDL-C in HIVInfected Individuals: ACTG 5087. HIV Clin Trials 2007; 8(1): 45-52.

16. Nauck M, Warnick GR, Rifai N. Methods for Measurement of LDL-Cholesterol: A Critical Assessment of Direct Measurement by Homogeneous Assays versus Calculation. Clinical Chemistry. 2002; 48(2): 236-254.

17. Mora S, Rifi N, Buring J, Ridker P. Comparison of LDL Cholesterol Concentrations by Friedewald Calculation and Direct Measurement in Relation to Cardiovascular Events in 27331 Women. Clinical Chemistry. 2009; 55(5): 888-94. doi:10.1373/clinchem.2008.117929..

18. Can M, Acikgoz S, Mungan G, Ugurbas E, Ankarali H, Sumbuloglu V, et al. Is direct method of low density lipoprotein cholesterol measurement appropriate for targeting lipid lowering therapy? International Journal of Cardiology 2010; 142(1): 105-107.

19. Timón-Zapata J, Laserna-Mendieta E, Pineda-Tenor D, Agudo-Macazaga M, Narros-Cecilia C, Rocha-Bogas MJ, et al. Extreme concentrations of high density lipoprotein cholesterol affect the calculation of low density lipoprotein cholesterol in the Friedewald formula and other proposed formulas. Clinical Biochemistry 2011; 44(17-18): 1451-6. doi: 10.1016/j.clinbiochem.2011.09.009. 